\title{
Anaphylaxis sugammadex-induced in a pediatric patient
}

\section{Anafilaxia por sugammadex en paciente pediátrico}

Juana Bautista Joyanes-Romo, ${ }^{1}$ Ana Isabel Navarro-Abad, ${ }^{2}$ José Miguel Urra-Ardanaz, ${ }^{2}$ Óscar González-Jiménez, ${ }^{1}$ Alberto Palacios, ${ }^{1}$

Jaime Vinicio Meneses-Sotomayor, ${ }^{1}$ Marta Pascual, ${ }^{3}$ Ma. de Gracia Villanueva, ${ }^{3}$ Davinia Garrido, ${ }^{4}$ Ana Joyanes, ${ }^{5}$ Pedro Ángel Galindo-Bonilla ${ }^{1}$

\begin{abstract}
Introduction: Sugammadex is a cyclodextrin that reverses neuromuscular blockade, especially of rocuronium. The occurrence of anaphylaxis produced by its use is of 1:1000 and 1:20000; it is observed mainly in subjects of Asian origin.

Case report: A 9-year-old boy of Asian origin who, after the administration of sugammadex, immediately manifested an episode of anaphylaxis, which was reverted by using adrenaline and antihistamines. The serum tryptase at two hours was $27.7 \mu \mathrm{g} / \mathrm{L}$; at 6 weeks, it was $3 \mu / \mathrm{L}$. The sugammadex $100 \mathrm{mg} / \mathrm{mL}$ skin test was positive. The basophil activation test was positive with sugammadex $20 \mathrm{mg} / \mathrm{mL}$.

Conclusion: The temporal relationship between the administration of the drug, the clinical manifestations, the elevation of tryptase, and the diagnostic tests performed, disclosed the episode of anaphylaxis associated with hypersensitivity to sugammadex.
\end{abstract}

Key words: General anesthesia; Sugammadex; Anaphylaxis; Basophil activation test; Pediatrics

\section{Resumen}

Introducción: Sugammadex es una ciclodextrina que revierte el bloqueo neuromuscular, especialmente de rocuronio. La incidencia de anafilaxia producida por su uso es de 1:1.000 y 1:20.000, se observa principalmente en sujetos de origen asiático.

Reporte de caso: Niño de 9 años, de raza asiática que tras la administración de sugammadex, inmediatamente manifestó un episodio de anafilaxia, la cual revirtió con el uso de adrenalina y antihistamínicos. La triptasa sérica a las 2 h fue de $27.7 \mu \mathrm{g} / \mathrm{L}$; a las 6 semanas fue $3 \mu \mathrm{g} / \mathrm{L}$. La prueba cutánea a sugammadex $100 \mathrm{mg} / \mathrm{mL}$ fue positiva. La prueba de activación de basófilos fue positiva con $20 \mathrm{mg} / \mathrm{mL}$ sugammadex. Conclusión: La relación temporal de la administración del medicamento, las manifestaciones clínicas, la elevación de la triptasa y las pruebas diagnósticas realizadas, identificaron el episodio de anafilaxia asociado con hipersensibilidad por sugammadex.

Palabras clave: Anestesia general; Sugammadex; Anafilaxia; Prueba de activación del basófilo; Niños

${ }^{1}$ Hospital General Universitario de Ciudad Real, Sección de Alergia, Ciudad Real, España ${ }^{2}$ Hospital General Universitario de Ciudad Real, Inmunología, Ciudad Real, España ${ }^{3}$ Hospital General Universitario de Ciudad Real, Servicio de Anestesia, Ciudad Real, España ${ }^{4}$ Departamento de Enfermería, Hospital General Universitario de Ciudad Real, Ciudad Real, España 5Universidad de La Laguna, Escuela de Medicina, Tenerife, España
Correspondencia: Juana Bautista Joyanes-Romo. jjoyanesromo@gmail.com

Recibido: 21-02-2021

Aceptado: 2021-07-25

DOI: $10.29262 /$ ram.v658i2.876 


\section{Introducción}

Sugammadex (SG) es una $\gamma$-ciclodextrina que se usa para revertir el efecto de los bloqueadores neuromusculares aminoesteroideos, con mayor afinidad por el rocuronio (RC). En comparación con la neostigmina, revierte el bloqueo rápida y predeciblemente, con pocos efectos secundarios de tipo colinérgicos. Encápsula al RC al disminuir sus niveles plasmáticos, esto crea un gradiente negativo y hace que difunda desde la unión neuromuscular hacia el plasma. ${ }^{1}$

SG es ampliamente utilizado en Japón, sin embargo, recientemente se ha observado un incremento en el número de casos de hipersensibilidad. ${ }^{2} \mathrm{Al}$ menos dos estudios informaron de hipersensibilidad y anafilaxia inducida por $\mathrm{SG}^{1,3}$ con una incidencia de hipersensibilidad del $5 \%$ y de anafilaxia del $0.3 \%$ después de usar una dosis única. ${ }^{3}$ Una encuesta mundial realizada entre marzo de 2016 y mayo de 2017 arrojó una incidencia estimada de anafilaxia de 1:1.000-1:20.000. ${ }^{4}$

El mecanismo exacto de esta hipersensibilidad es desconocido. Las ciclodextrinas pueden sensibilizar fácilmente ya que se emplean en la fabricación de productos alimenticios y cosméticos. Los estudios de $\mathrm{Min}^{1}$ y $\mathrm{Kam}^{5}$ no pudieron establecer una correlación entre la hipersensibilidad y niveles séricos de triptasa, pruebas cutáneas (PC) o anticuerpos inmunoglobulina (Ig) E o IgG específicos de SG. Se ha propuesto que el complejo RC-SG en sí mismo puede provocar una reacción alérgica debido a un cambio en sus propiedades inmunológicas. ${ }^{6}$

Presentamos un caso de anafilaxia por SG en un niño de origen chino, donde las $\mathrm{PC}$ y la prueba de activación de basófilos (PAB) fueron determinantes para establecer el diagnóstico.

\section{Caso clínico}

Niño de 9 años de origen chino con historial de padecer rinoconjuntivitis estacional, sin alergias a medicamentos, ni otros antecedentes de interés. Al realizarle una apendicectomía y tras la administración de $2 \mathrm{mg} / \mathrm{kg}$ de $\mathrm{SG}$ para revertir el efecto bloqueador del RC, presentó hipotensión, broncoespasmo, enrojecimiento facial y edema de los labios y los párpados. Las manifestaciones revirtieron rápidamente con el uso de adrenalina intramuscular y antihistamínicos. Durante la cirugía, se le había administrado: paracetamol, ketorolaco, amoxicilina/ácido clavulánico, ondansetron, dexametasona, metilprednisolona, midazolam, propofol, fentanilo, RC y SG. En ese momento se le realizó una determinación de triptasa sérica $(19.4 \mu \mathrm{g} / \mathrm{L})$ y a las dos horas $(27.7 \mu \mathrm{g} / \mathrm{L})$.

A las 6 semanas se realizó la siguiente evaluación alergológica: determinación de $\operatorname{IgE}$ total $(23.3 \mathrm{kU} / \mathrm{L})$ y triptasa sérica $(3 \mu \mathrm{g} / \mathrm{L})$, prueba de punción cutánea y intradermorreacción según técnica habitual y prueba de exposición controlada con aquellos fármacos usados en el acto perianestésico con los que se podían hacer. Asimismo, se realizó un PAB con la ampolla de SG (Bridion $\mathbb{R} 100 \mathrm{mg} / \mathrm{mL}$ a una concentración de $20 \mathrm{mg} / \mathrm{mL}$ ), con RC (Esmerón ${ }^{\circledR} 10 \mathrm{mg} / \mathrm{mL}$ ) y con su mezcla 1:1, utilizando el kit comercial Glycotope de Biotechnology con lectura por citometría de flujo FacScan de Beeton Dickinson según técnica descrita, ${ }^{7}$ se considera activación positiva cuando encontramos más de un $15 \%$ de señal.

Los resultados de la prueba de punción cutánea fueron positivos para $\mathrm{SG} 100 \mathrm{mg} / \mathrm{mL}(6 \times 5 \mathrm{~mm})$ y mezcla $1: 1$ $\mathrm{SG} / \mathrm{RC}(5 \times 4)$, y negativos para RC $10 \mathrm{mg} / \mathrm{mL}$. Histamina $5 \times 5 \mathrm{~mm}$.

El PAB con $\mathrm{SG} 20 \mathrm{mg} / \mathrm{mL}$ resultó positivo y negativo con $\mathrm{RC} 10 \mathrm{mg} / \mathrm{mL}$ y con mezcla $1: 1 \mathrm{de} \mathrm{SG} / \mathrm{RC}$.

Las PC y las pruebas de exposición con el resto de los medicamentos fueron negativos y con buena tolerancia a los mismos.

La Figura 1 muestra los resultados de las pruebas de prick a RC y SG.

\section{Discusión}

En los niños, una recuperación rápida de la anestesia se puede acompañar de tos, laringoespasmo e hipoxemia, lo que puede llegar a confundirse con una reacción anafiláctica. ${ }^{8}$ En nuestro paciente, el nivel elevado de triptasa de forma inmediata y a las dos horas, las PC positivas y el PAB mostraron que el paciente había presentado un episodio de anafilaxia asociado con hipersensibilidad a SG.

En los pacientes que presentan reacciones de hipersensibilidad tras la administración de $\mathrm{SG}$, las reacciones descritas más frecuentemente son erupción cutánea (80\%), hipotensión $(60 \%)$, taquicardia (53\%) o desaturación $(47 \%) .{ }^{1}$ Los pacientes tratados con SG en comparación con neostigmina, presentan una incidencia menor de efectos adversos como bradicardia, náuseas, vómitos y signos generales de parálisis residual posoperatoria, sin embargo, presentan una incidencia mayor de anafilaxia. ${ }^{2}$

Al comparar la incidencia de anafilaxia entre Japón y Reino Unido, al ser más frecuente su aparición en pacientes de origen asiático, se plantearon la posibilidad de que exista una predisposición a presentar anafilaxia por SG en asiáti$\cos ^{2}$ Nuestro paciente es un niño de raza asiática.

Se han observado reacciones alérgicas durante la primera administración de SG con PAB falsamente positivo cuando la ampolla de SG ha sido expuesta a la luz, lo que sugiere que un compuesto desnaturalizado sea el responsable de la hipersensibilidad. ${ }^{9}$ Nosotros tuvimos especial cuidado en proteger la ampolla de SG antes de realizar las PC y el PAB. Otra posibilidad de que pueda aparecer anafilaxia tras la primera administración, sería que los pacientes se hubieran sensibilizado previamente a ciclodextrinas contenidas en alimentos o cosméticos. ${ }^{10}$ Este podría ser el origen de la sensibilización en nuestro paciente.

Se ha descrito que el complejo SG-RC puede provocar la respuesta alérgica debido a un cambio conformacional en un paciente que no es alérgico a los componentes por separado. ${ }^{6}$ En nuestro caso, el PAB con una mezcla de SG-RC fue nega- 
tivo en el límite de positividad, mientras que fue positivo con SG exclusivamente, las PC fueron positivas con SG, pero negativas con la combinación, lo que indica que el fármaco implicado en la anafilaxia fue SG. Durante el acto perianestésico se utilizan múltiples medicamentos que pueden ser la causa de la reacción anafiláctica; vale la pena mencionar que con algunos de ellos no se pueden hacer pruebas de exposición por razones obvias (relajantes musculares, inductores de la anestesia, entre otros). En nuestro caso descartamos la participación del resto de medicamentos en el origen de la anafilaxia. En España, se informó de un paciente adulto con anafilaxia inducida por SG diagnosticada por la clínica y las pruebas cutáneas; en nuestro caso, además, realizamos un PAB que apoyó el diagnóstico. Otros estudios han comunicado casos de pacientes con hipersensibilidad a SG en los que demostraron la utilidad del PAB. ${ }^{2,9}$

\section{Conclusión}

Presentamos un episodio de anafilaxia asociado con SG en un niño de origen asiático. Donde las PC y el PAB fueron determinantes para confirmar el diagnóstico. Destacamos el PAB como técnica diagnóstica en sospecha de alergia a SG al evitar la realización de pruebas in vivo, con el riesgo que esto representa para el paciente.

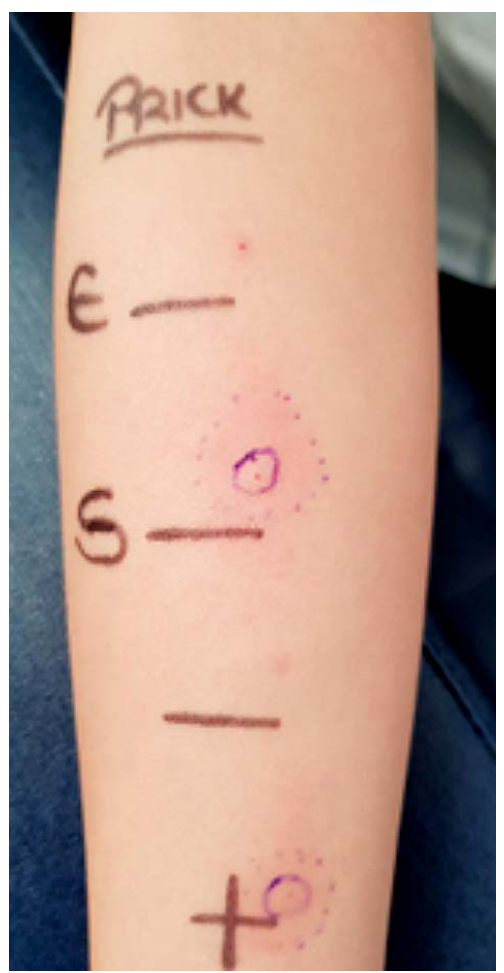

Figura 1. Prueba de punción cutánea con sugammadex (S) a $100 \mathrm{mg} / \mathrm{mL}$. Con rocuronio (E) a $10 \mathrm{mg} / \mathrm{mL}$, suero salino (-) e histamina (+) a $10 \mathrm{mg} / \mathrm{mL}$

\section{Referencias}

1. Min KC, Bondiskey P, Schulz V, Woo T, Assaid C, Yu W, et al. Hypersensitivity incidence after sugammadex administration in healthy subjects: a randomised controlled trial. $\mathrm{Br} J$ Anaesth. 2018;121(4):749-757. DOI: 10.1016/j.bja.2018.05.056

2. Orihara M, Takazawa T, Horiuchi T, Sakamoto S, Nagumo K, Tomita Y, et al. Comparison of incidence of anaphylaxis between sugammadex and neostigmine: a retrospective multicentre observational study. Br J Anaesth. 2020;124 (2):154-163 DOI: 10.1016/j.bja.2019.10.016

3. Savic L, Savic S, Hopkins PM. Sugammadex: the sting in the tail? Br J Anaesth. 2018;121(4):694-697. DOI: 10.1016/j.bja.2018.07.014

4. Jabaley CS, Wolf FA, Lynde GC, O'Reilly-Shah V. Crowdsourcing sugammadex adverse event rates using an in-app survey: feasibility assessment from an observational study. Ther Adv Drug Saf. 2018;9(7):331-342. DOI: 10.1177/2042098618769565

5. de Kam PJ, Nolte H, Good S, Yunan M, Williams-Herman DE, Burggraaf $J$, et al. Sugammadex hypersensitivity and underlying mechanisms: a randomised study of healthy nonanaesthetised volunteers. $\mathrm{Br} J$ Anaesth. 2018;121(4):758-767. DOI: 10.1016/j.bja.2018.05.057
6. Baldo BA. Anaphylaxis caused by sugammadex- rocuronium inclusion complex: what is the basis of the allergenic recognition? J Clin Anesth. 2019;54:48-49. DOI: 10.1016/j.jclinane.2018.10.017

7. Urra JM, Pérez-Lucendo I, Extremera A, Feo-Brito F, Alfaya T. The Method for selecting basophils might be determinant in the basophil activation test in patients with mastocytosis. J Investig Allergol Clin Immunol. 2020;30(1):65-67. DOI: 10.18176/jiaci.0442

8. Shen X, Hu C, Li W. Tracheal extubation of deeply anesthetized pediatric patients: a comparison of sevoflurane and sevoflurane in combination with low-dose remifentanil. Paediatr Anaesth. 2012;22(12):1179-1184. DOI: 10.1111/j.1460-9592.2012.03906.x

9. Yamada T, Suzuki T, Murase R, Nagata H, Kosugi S. Anaphylactic reactions to native and light-exposed sugammadex suggested by basophil activation test: a report of 2 cases. A A Pract. 2018;11(7):181183. DOI: 10.1213/XAA.0000000000000774

10. Soria A, Motamed C, Gaouar H, Chemam S, Amsler E, Francès C. Severe reaction following sugammadex injection: hypersensitivity? J Investig Allergol Clin Immunol. 2012;22(5):382. Disponible en: http:// www.jiaci.org/issues/vol22issue5/8-20.pdf

ORCID

Juana Bautista Joyanes-Romo,0000-0002-4719-8291; Ana Isabel Navarro-Abad, 0000-0002-4861-5704;

José Miguel Urra-Ardanaz, 0000-0003-3059-7429; Oscar González-Jiménez,0000-0003-4798-154X; Alberto Palacios, 0000-0002-7180-8539; Jaime Vinicio Meneses-Sotomayor, 0000-0002-9452-3317; Marta Pascual, 0000-0002-9677-0792;

Ma. de Gracia Villanueva 0000-0003-0071-7629; Davinia Garrido, 0000-0003-2700-4565; Ana Joyanes, 0000-0002-3365-4129;

Pedro Ángel Galindo-Bonilla, 0000-0002-1296-6748 